\title{
Disc Motor: Conventional and Superconductor Simulated Results Analysis
}

\author{
David Inácio ${ }^{1}$, João Martins ${ }^{1}$, Mário Ventim Neves ${ }^{1}$, Alfredo Álvarez², \\ and Amadeu Leão Rodrigues ${ }^{1}$ \\ ${ }^{1}$ CTS/UNINOVA, Faculty of Sciences and Technology - Universidade Nova de Lisboa, \\ Quinta da Torre, 2829-516 Caparica, Portugal \\ ${ }^{2}$ Departament of Electrical Engineering, Escuela de Ingenierias Industriales, Universidad de \\ Extremadura, E-06006 Badajoz, Spain
}

\begin{abstract}
Taking into consideration the development and integration of electrical machines with lower dimensions and higher performance, this paper presents the design and development of a three-phase axial flux disc motor, with $50 \mathrm{~Hz}$ frequency supply. It is made with two conventional semi-stators and a rotor, which can be implemented with a conventional aluminum disc or a high temperature-superconducting disc. The analysis of the motor characteristics is done with a 2D commercial finite elements package, being the modeling performed as a linear motor. The obtained results allow concluding that the superconductor motor provides a higher force than the conventional one. The conventional disc motor presents an asynchronous behavior, like a conventional induction motor, while the superconductor motor presents both synchronous and asynchronous behaviors.
\end{abstract}

Keywords: Axial disc motor; Superconductivity; HTS; YBCO; Finite elements program.

\section{Introduction}

The development and research of lower size electrical machines with higher performance has become a fundamental issue in the electrical machines community. With applications in many systems, the development and improvement of traction electrical motors presents one of the nowadays biggest challenges. Being the automobile the principal means of transport used in the world, they have deserved higher attention. Some countries, such as Portugal, promote and ensure financial advantages to users that acquire electrical vehicles. The integration of electrical motors in the active production of movement in a vehicle, instead of fuel motors, is the main challenge face to the various automobile's constructors, forcing them to establish lines of ecological vehicles, hybrids and fully electric, essential for environmental preservation. The electrical motors development is also "forced" by the increasing fuel prices and by the $\mathrm{CO}_{2}$ limited emissions, imposed by the European Commission. These actions and incentives were the starting point for a all new ecological journey, where the simultaneous use of combustion and electrical motors makes possible the simultaneous reduction of $\mathrm{CO}_{2}$ emissions and fuel consumption, desired in the actual economical crisis. On the other hand the production of fully electric cars has also become an 
important objective integrating, for the example, the use of hydrogen based power supplies [1][2] or renewable energies, such as photovoltaic [3].

The discovery of high temperature superconductor - HTS - materials along with their characteristics [4], has improved the development and integration of superconducting electric motors and superconductivity in various systems replacing conventional motors. Those superconducting motors are applied in different systems: measurement instruments (Superconducting Quantum Interference Devices [5]), electronics [6], train motors and technology [7], ships [8], vehicles [9] and small airplanes [10]. The use of HTS materials provides the achievement of smaller and lighter electrical vehicles. Fuel cells and hydrogen technology can also be integrated, where the low temperature of liquid hydrogen can cool down the HTS elements of the traction motor, making them superconductors. This integration can rise some security aspects that must be overcome with the correct development and improvement of such systems, in order to ensure the safety of users of these vehicles [11].

\section{Contributions to Technological Innovation}

This paper compares the performance of two disc motors: a conventional one and a HTS one. From the obtained results, one can observe that the superconductor motor develops a higher force than its equivalent aluminum motor. Furthermore, a technological important feature, the HTS motor present synchronous behavior while the conventional present asynchronous behavior.

\section{Superconductivity}

The high temperature superconductor - HTS - materials present zero resistance only in stationary conditions. In alternate field applications HTS material presents losses. The HTS materials are subject to penetration of quantized flux within the superconductor sample through the cores of current vortices [13]. These vortices consist of a magnetic flux cylindrical core aligned with the applied magnetic field, with a distance equal to the coherence length $\xi$. This core is surrounded by a cylinder of supercurrents that flow in a vortex ring with a thickness equal to the London penetration depth $\lambda$.

The vortices are distributed in a cantered hexagonal geometry, as presented in Fig. 1, which appears in the superconductor from the edges to the centre. The vortices

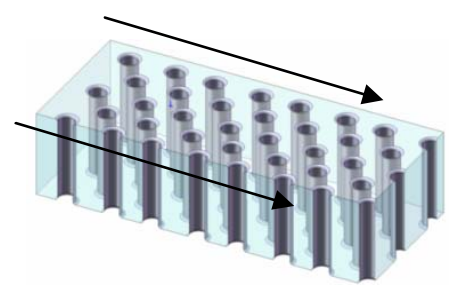

Fig. 1. Vortices dynamics in a superconductor sample 
are subject to the Lorentz force that tends to move them. Accordingly to Maxwell Law, an electric field (1) is induced due to the magnetic field variation inside the vortices within the superconductor.

$$
\vec{E}=\vec{B} \times \vec{v}
$$

In (1), $\vec{B}$ denotes the magnetic field and $\vec{v}$ the vortices displacement speed. Current densities $\mathbf{J}$ are induced in HTS material and there are resistive losses, proportional to the product of the induced current density by the electric field created. According to Barnes [25], these losses are proportional to torque by a factor of $2 \pi$. For pure superconductor materials the variation of applied magnetic field in a superconductor makes the vortices traveling freely by the sample. When the superconducting materials present impurities or defects, the vortices are subject to the pinning flux, the vortices stay trapped in impurities or imperfections (pinning centers) and the magnetic behavior of the sample depends on its magnetic history, independent of time. For higher pinning is need higher current density to Lorentz force win the pinning force, causing more losses and torque.

\section{Electric Disc Motors}

The first developed electrical machine was the primitive axial flux machine, built by Faraday. Since then, the constant development of radial flow electric machines has made it to prevail on axial flow, and around 1900 was already used in large scale. At that time, the study and development of electric machines was influenced by the materials and manufacturing processes available. Even before 1980 the axial flux machinery begun to have great applicability in low speed applications. The expensive production of axial flux induction motors was a factor that limited the use of such motors, such as the fact that the faulty construction of the rotor limited the speed of the machine [12].

Today is possible to build axial flux motors with relatively low weight, low volume and with excellent mechanical and dynamic performance, which makes the axial flux induction machine a solution in several applications.

Disc motors using HTS materials have been used mainly in two topologies: disc motors where the rotor elements of superconducting materials act as magnets [14] or motors where the rotor is a single superconductor disc presenting hysteresis behaviour composed, for example, by YBCO [15] or BSCCO [16].

With the first topology the motor presents behaviour similar to a synchronous motor, while with the second one presents a more complex behaviour. Experimental results for a rotoric ring (cylinder) [17] or for a disc [18] in the HTS hysteresis motors show this dual behaviour. This does not happen when one has a rotoric ring with low electrical conductivity, as in ferromagnetic materials, due to the characteristics of pinning that HTS materials present and due to the dynamics of vortices in this type of material [19]. The stators of the disc motor can be conventional ones, composed by copper windings and steel, or superconductor ones, when high current densities are required [19]. 
The disc motors have been elected to be used in electric vehicles that use fuel-cell technology [20]. The liquid hydrogen used as fuel cells, could also be used [11] to cool the HTS materials in the rotor disc of the motor installed on the electric vehicle.

Currently there are essentially three categories of machines optimized with superconductor materials: hysteresis, reluctance and trapped field motors [21], [22], [23]. Theoretically, the torque produced from an HTS machine is three to five times higher than the torque obtained for the same conventional machine, made by conventional materials [24].

\section{Developed Motor}

\subsection{Topology}

The built disc motor is composed by two semi-stators with 24 slots and 24 conventional cooper windings each one, a steel shaft, a rotor consisting of an aluminum disc or a YBCO disc, two support bearings and fixing screws. Fig. 2 shows the built motor.

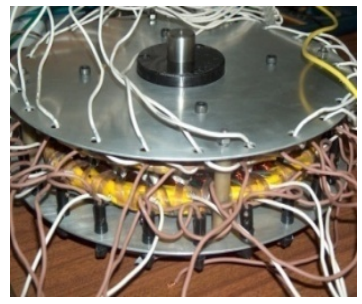

Complete motor.

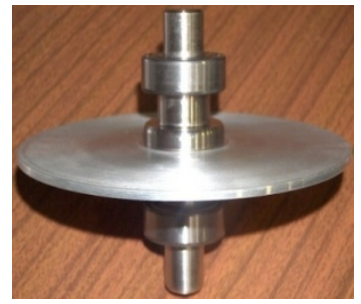

Conventional rotor with shaft.

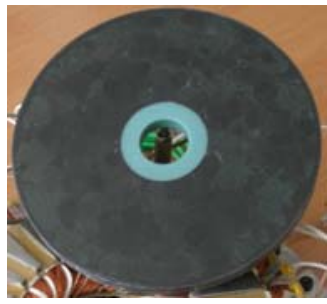

Superconductor rotor.

Fig. 2. Built disc motor

The spacing between the semi-stators and rotor should be as small as possible to assure lower dispersion in the flux produced by the coils of semi-stators, ensuring a higher torque. In the mechanical connection between the semi-stator paramagnetic material (mica) high magnetic reluctance screws were used, in order to cancel the magnetic connection between semi-stators. A system that ensures the adjustment nuts of airgap was also integrated.

\subsection{Characteristics}

This motor, when the rotor is composed of aluminum, has an asynchronous behavior, functioning as a conventional induction motor.

The operation of this motor is quite similar to the drum induction motor, where the flux, coming from one semi-stator, penetrates the rotor disc perpendicularly and closes itself onto the other semi-stator. The disc motor in study has the electrical circuit on the side of the stator, with 24 independent coils, and the rotor consists of an aluminum disc. The stator coils create a magnetic rotating field making the aluminum rotor "feel" a variable inductive magnetic field. Thus, electromotive forces will be 
induced on the disc giving origin to currents in the rotor disc. The induced currents produce an electromagnetic torque, $T_{\text {elect }}$, which places the rotor to rotate.

When the rotor is composed of HTS materials it presents a complex behavior, showing both synchronous and asynchronous behaviors [25]. At startup, the stator rotating field magnetizes the rotor HTS materials and, in this rotor magnetization process, vortices are induced in the HTS material. These vortices traveling in the HTS disc and can be subject to pinning centers, that pinning them, magnetizing the rotor and creating a field in the rotor that, in steady state, presents a constant phase relatively to the rotating field created by the stator. The interaction between these two fields origins a motor torque that presents an amplitude equal to the product of the amplitudes of two fields with the phase between them (2).

$$
T=B_{\max } \times H_{\max } \times \sin \left(\alpha_{\mathrm{B}} \wedge \alpha_{\mathrm{H}}\right) .
$$

\subsection{Simulations}

The motor described above was simulated with a commercial finite elements software package, FLUX2D®. In this package, the non-HTS material parameterization was made with same materials already included, like copper, aluminum and steel. In this program, the material parameterization was made with same materials that were parameterized, like the copper, the aluminum and the steel. For the HTS materials, namely $\mathrm{YBCO}$, the parameterization was based using the E-J power law (3).

$$
\rho(E, B)=\frac{E_{c}^{\frac{1}{n(B)}}}{J_{c}(B)}|E|^{\frac{n(B)-1}{n(B)}}+\rho_{0},
$$

$J_{\mathrm{c}}$ denotes the critical current density, $E_{\mathrm{c}}$ the critical electric field, $n$ the exponent and $\rho_{0}$ the resistivity. The following parameter values, available from the used software package, were used for the HTS material (YBCO): $E_{\mathrm{c}}=10^{-4} \mathrm{~V} / \mathrm{m}$ (Critical Electric Field); $J_{\mathrm{c} 0}=4,4 \times 10^{6} \mathrm{~A} / \mathrm{mm}^{-2}$ (Critical Current Density); $B_{0}=10^{6} \mathrm{~T}$ (Magnetic Induction Field); $n_{0}=15$ (Exponent); $\rho_{0}=10^{-13} \Omega \mathrm{m}$ (Additional resistivity).

The analysis of the motor characteristics is done with FLUX2D®, being the modeling performed as a linear motor. The obtained results are presented below. The flux lines resulting from the supply of the coils in the stator are shown in Fig. 3. It can be seen that, for a linear stator with identical dimensions to the cylindrical motor designed, two magnetic poles are created magnetizing the rotor.

The current density distribution analysis over the airgap shows, as expected, a sinusoidal distribution with two poles, as presented in Fig. 4.

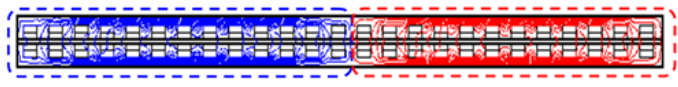

Fig. 3. Flux lines produced by the semi-stators supply 


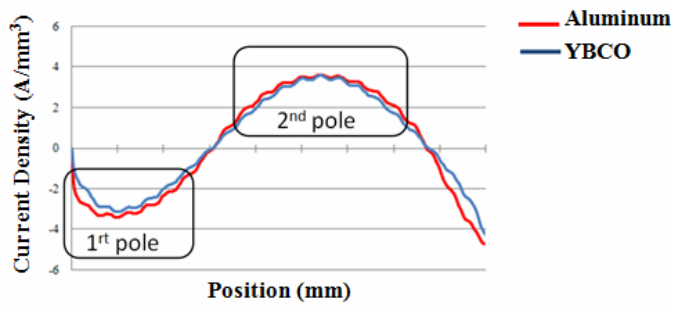

Fig. 4. Simulated current density distribution over the airgap

The results presented in Fig. 5 and Fig. 6 allows concluding that the motors present the expected behavior. Fig. 5 shows the force, for both motors in a no-load condition. The motor with the aluminum rotor presents an asynchronous behavior.

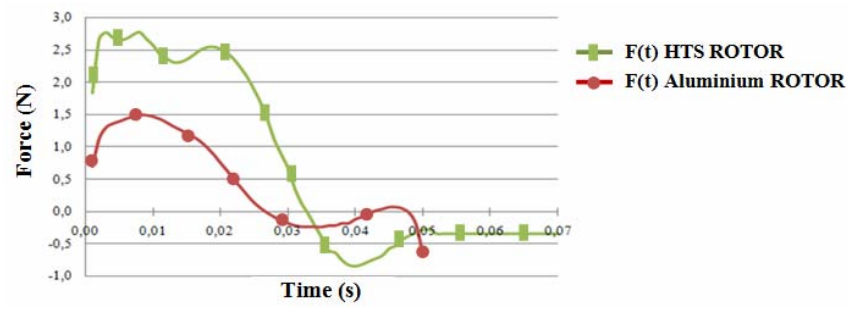

Fig. 5. Comparison of longitudinal force time evolution between the simulated obtained characteristics for motors without load

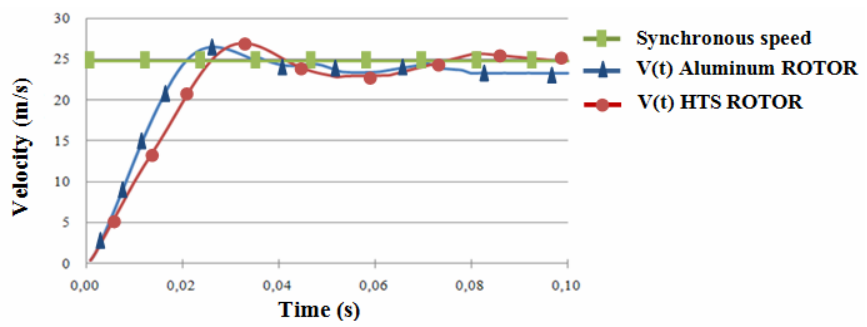

Fig. 6. Comparison of longitudinal force time evolution between the simulated obtained characteristics for motors without load

In Fig. 6 is possible to observe that the aluminum rotor motor, in steady state, does not achieve synchronous linear speed (approximately $25 \mathrm{~m} / \mathrm{s}$ for an effective length stator of $0,495 \mathrm{~m}$, a frequency of $50 \mathrm{~Hz}$ and 2 poles). The HTS rotor motor behaves like a synchronous motor. In steady state, as shown in Fig. 6, it can be seen that it does achieve synchronous speed, behaving as an asynchronous motor when not in steady state. Two important characteristics of the HTS motor can be observed in above figures. The HTS linear motor presents a higher force than the conventional aluminum motor and also presents dual synchronous and asynchronous behavior. 
Running this motor from start up to steady state, it behaves like an asynchronous motor, working with slip until it reaches the steady state. After reaching the steady state condition the rotor rotates with synchronous speed, acting like a permanent magnet motor.

\section{Conclusions and Future Work}

From the obtained results it is possible conclude that the conventional disc motor behaves like an induction motor, with asynchronous behavior while the HTS disc motor presents asynchronous and synchronous behaviors. During asynchronous regime, the magnetization of the superconductor, by the rotating field created in the stator, induces currents in superconducting materials, which establish a magnetic flux that, reacting with the stator flux give rise to an electromagnetic torque, proportional to the losses. In steady state operation, the motor torque is null due the synchronism between the field created by the stator and the field resulting in the rotor. Both fluxes are synchronous thus no flux variation exists and no losses are created.

From the Flux $2 D \circledR$ obtained results it can be seen that the HTS motor presents a higher force than the equivalent aluminum motor. However, the obtained force in the simulated HTS disc motor has not been as superior as in the aluminum disc motor. Possible causes may be the simplified simulation problem, a godless accurate parameterization of the materials in the finite element package and the use of a simulated linear motor instead a rotary motor.

Future work considers improving the material parameters in the finite elements program, and develops a more realistic simulation of this linear motor in order to obtain an approach of the electromagnetic characteristic of disc motor. Another approach is the study of this motor considering the load and/or speed variation.

Acknowledgements. Authors would like to thank CTS (Centre for Technology and Systems) of UNINOVA (Institute of New Technologies) for financial supporting this work.

\section{References}

1. Barclay, F.: Fuel Cells, Engines and Hydrogen. Wiley, Chichester (2006)

2. Thomas, C., James, B., Lomax Jr., F., Kuhn Jr., I.: Fuel options for fuel cells vehicle: hydrogen, methanol or gasoline? International Journal of Hydrogen Energy 25, 551-567 (2000)

3. Solar electrical vehicles information, http: / /www. solarelectricalvehicles.com

4. Superconductivity information, http://superconductors .org/

5. Casas, J., Miyamoto, N., Nakane, H., Goto, E.: Performance of a double SQUID Magnometer. IEEE Transactions on Magnetics 27(2) (1991)

6. Van Duzer, T.: Superconductor Electronic Device Applications. IEEF Journal of Quantum Electronics 15(II) (1989)

7. MAGLEB Official Website information, http://www. smtdc.com 
8. Snitchler, G., Gamble, B., Kalsi, S.: The performance of a 5 MW high temperature superconductor ship propulsion motor. IEEE Transactions on Superconductivity 15(2) (2005)

9. Sumitomo Electric information, http://global-sei.com/super/topics_e/index.html

10. Electromechanical Systems Applied Superconductivity information, http: / / www . masbret. com/UAPT.html

11. Thomas, C., James, B., Lomax Jr., F., Kuhn Jr., I.: Int. Journal of Hydrogen Energy 25(6, 1), 551-567 (2000)

12. Axial flux technology information, http: //www.axcomotors.com/axial-flux_technology.html

13. Abrikosov, A.: Zh. Eskp. Teor. Fiz. 35, 1442; Sov. Phys. JETP 5, 1174 (1957)

14. Marquez, I., Granardos, X., Obradors, X., Pallares, J., Bosch, R.: IEEE Transactions of Applied Superconductivity 9(2), 1249-1252 (1999)

15. Álvarez, A., Suárez, P., Cáceres, D., Granados, X., Péres, B., Ceballos, J.: Disk-shaped superconducting rotor for an axial flux induction motor. Physica C 398, 157-160 (2004)

16. Nakamura, T., Jung, H., Muta, I., Hoshino, T.: Synchronization of an axial-type Bi-2223 bulk motor operated in liquid nitrogen. Supercond. Sci. Technol. 17(11), 1319-1323 (2004)

17. Tsuboi, Y., Ohsaki, H.: Torque characteristics of a motor using bulk superconductors in the rotor in the transient phase. IEEE Trans. Applied Supercond. 13, 2210-2213 (2003)

18. Álvarez, A., Suárez, P., Cáceres, D., Cordero, E., Ceballos, J., Péres, B.: Disk-shaped Superconducting Rotor under a Rotating Magnetic Field: Speed Dependence. IEEE Trans. Applied Supercond. 15(2), 2174-2177 (2005)

19. Álvarez, A., Suárez, P., Cáceres, D., Granados, X., Obradors, X., Bosch, R., Cordero, E., Péres, B., Caballero, A., Blanco, J.: Superconducting armature for induction motor of axial flux based on YBCO bulks. Physica C 372-376(3), 1517-1519 (2002)

20. Rahman, K., Patel, N., Ward, T., Nagashima, J., Caricchi, F., Crescimbini, F.: Application of Direct Drive Wheel Motor for Fuel Cell Electric and Hybrid Electric Vehicle Propulsion System. In: Conference Record IEEE 39th Industry Application Conference, vol. 3 (2004)

21. Match, L.: Electromagnetic an Electromechanical Machines. Wiley \& Sons, Chichester (1986)

22. McCulloch, M., Dew-Hughes, D.: Brushless Ac machines with high temperatures superconducting rotors. Material Sciences and Engineering 53(1-2), 211-215 (2000)

23. Barnes, G., McCulloch, M., Dew-Hughes, D.: Applications and Modelling of Bulk HTS in Brushless AC Machines. Supercond. Sci. Technol. 13, 875-878 (2000)

24. Bondrea, N., Leão Rodrigues, A.: Torque comparison of an eight-pole permanent magnet excited and a high temperature superconductor disc motor. In: Proceedings of the 4th International Workshop on Processing and Applications of Superconducting (RE)BCO Large Grain Materials PASREG 2003, Jena, Germany (2003)

25. Barnes, G.: Computational modelling for type-II superconductivity and the investigation of high temperature superconducting electrical machines, Ph.D. Thesis, Oxford Univ. (2000) 